\title{
A Tri-Layered Hydrogel Scaffold for Vocal Fold Tissue Engineering
}

R. Kevin Tindell ${ }^{1}$, Michael J. McPhail ${ }^{2}$, Cheryl Myers ${ }^{2}$, Juergen Neubauer ${ }^{2}$, Justin M. Hintze ${ }^{2}$, David G. Lott ${ }^{2,3^{*}}$, Julianne L. Holloway ${ }^{1 *}$

${ }^{1}$ Chemical Engineering; School for Engineering of Matter, Transport and Energy; Arizona State University; Tempe, AZ

${ }^{2}$ Head and Neck Regenerative Medicine Laboratory; Mayo Clinic Arizona; Scottsdale, AZ

${ }^{3}$ Division of Laryngology; Mayo Clinic Arizona; Phoenix, AZ

*Corresponding Authors: Julianne.Holloway@asu.edu (J.L.H), Lott.David@mayo.edu (D.G.L)

\begin{abstract}
The lamina propria within the vocal fold (VF) is a complex multi-layered tissue that increases in stiffness from the superficial to deep layer, where this characteristic is crucial for VF sound production. Tissue engineered scaffolds designed for VF repair must mimic the biophysical nature of the native vocal fold and promote cell viability, cell spreading, and vibration with air flow. In this study, we present a unique tri-layered, partially-degradable hydrogel scaffold that mimics the multi-layered structure of the VF lamina propria. Using thiol-norbornene photochemistry, tri-layered hydrogel scaffolds were fabricated via layer-by-layer stacking with increasing polymer concentration from the top to middle to deep layer. Mechanical analysis confirmed hydrogel modulus increased with increasing polymer concentration. Partially-degradable hydrogels promoted high cell viability and cell spreading in 3D as assessed via live/dead and cytoskeleton staining, respectively. Importantly, partially-degradable hydrogels maintained some degree of the 3D polymer network following protease exposure, while still enabling encapsulated cells to remodel their local environment via protease secretion. Finally, the tri-layered hydrogel scaffold successfully vibrated and produced sound in proofof-concept air flow studies. This work represents a critical first step towards the design of a multilayered, hydrogel scaffold for vocal fold tissue engineering.
\end{abstract}

\section{Keywords}

Tissue engineering, vocal folds, hydrogels, hyaluronic acid, multi-layered hydrogel 


\section{Introduction}

The larynx is located above the trachea and is critical for facilitating basic functions such as speaking, breathing, and prevention of aspiration. ${ }^{1}$ The vocal folds (VFs) are a functional unit of the larynx and are responsible for phonation (i.e. vibration to produce sound). Abnormalities in sound production are common disorders with an estimated $1 \%$ of the American population being affected by dysphonia (i.e. voice that sounds raspy, weak, breathy, etc.). ${ }^{2}$ VF scarring, one of the most common causes of dysphonia, can be a consequence of recurrent phonotrauma (e.g. yelling, throat-clearing, manipulating sound, etc.), radiation from cancer therapy, or local inflammatory responses after abuse or injury. ${ }^{3,4}$

A fundamental goal of voice restoration is repairing VF biomechanical properties to restore vibratory characteristics and phonation. ${ }^{5}$ The VFs contain a unique heterogenous structure with an external squamous epithelium and basement membrane, followed by the lamina propria, and the thyroarytenoid muscle. ${ }^{1}$ The most critical layer for phonation is the lamina propria, which consists of a tri-layered tissue that increases in stiffness from the superficial layer to the deep layer (Figure 1). ${ }^{6,7} \mathrm{VF}$ scarring is characterized by increased deposition of disorganized collagen and a reduction in hyaluronic acid (HA). HA is the most abundant glycosaminoglycan in the VF and is critical for phonation. ${ }^{8-10}$ These compositional changes increase VF stiffness and, correspondingly, impede phonation. ${ }^{8,11}$ Common treatment options for repairing scarred VFs (e.g. fat, collagen, or hyaluronic acid injection; scar dissection; etc.) do not sufficiently restore the native VF structure. ${ }^{4,12}$ Thus, alternative strategies focused on repairing the VF structure, particularly the lamina propria, are needed to adequately restore tissue function.

Tissue engineering is a promising approach that combines medicine, biology, and engineering to regenerate damaged or diseased tissue. ${ }^{13}$ Tissue engineering strategies that regenerate functional VF tissue following scarring would enable restoration of a normal voice to a previously aphonic or dysphonic patient. ${ }^{1}$ Ideally, VF tissue-engineered scaffolds would vibrate at physiological conditions, promote cell integration and spreading, and regenerate functional VF tissue. Xenogenic scaffolds using the porcine small intestine submucosa have been observed to improve VF tissue regeneration and vibratory function in vivo. ${ }^{14}$ However, xenogenic scaffolds may induce an undesirable 
immunogenic response after implantation. New biocompatible scaffolds that mimic the VF's heterogeneous properties are needed to sufficiently promote tissue regeneration.

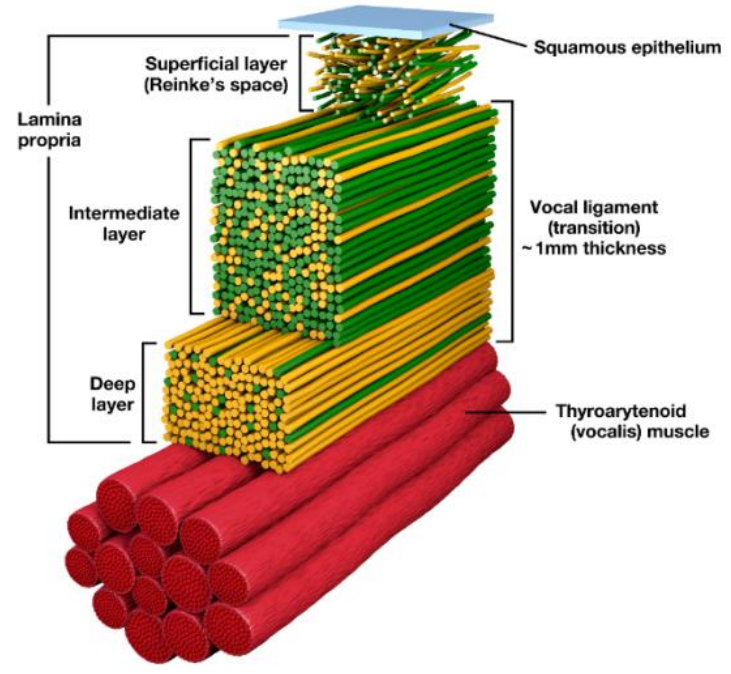

Figure 1: Diagram of the vocal fold lamina propria. The lamina propria contains three layers: superficial, intermediate, and deep. Elastin and collagen fibers are shown in green and yellow, respectively. The underlying thyroarytenoid muscle is shown in red. ${ }^{1}$ (Reprinted from ref. 1 with permission from Elsevier.)

A promising approach is to use scaffolds composed of extracellular matrix (ECM) components, such as collagen and $\mathrm{HA}$, which can be formulated to match the mechanical properties of the VF lamina propria. ${ }^{15-17}$ In particular, HA is advantageous due to its natural bioactivity and ability to promote physiological vibration. ${ }^{7,10,18,19}$ However, non-crosslinked HA has a rapid degradation rate within the VF due to the presence of hyaluronidase.$^{10}$ To improve HA stability, HA is modified with reactive functional groups to allow crosslinking into highly tunable three-dimensional (3D) hydrogels. ${ }^{11}$ For example, norbornene-modified hyaluronic acid (NorHA) reacts with thiol functional groups in the presence of a photoinitiator and light exposure. ${ }^{20}$ Norbornene-thiol chemistry can be used to either crosslink HA using a bifunctional crosslinker or tether biomolecules to HA via a monofunctional molecule. ${ }^{21}$ An ideal hydrogel for VF tissue engineering would be partially-degradable, where nondegradable crosslinks provide mechanical integrity over time for vibration and degradable crosslinks enable cells to spread, migrate, and regenerate new tissue. The specific degradation mechanism (e.g. hydrolytic, proteolytic, etc.) and kinetics can be tuned through the use of different types of crosslinkers and functional groups. Of particular interest, HA hydrogels crosslinked with a proteolyticallydegradable peptide allows nearby cells to degrade their local environment and spread in 3D through protease secretion (e.g. matrix metalloproteases (MMPs)). ${ }^{22-25}$ 
Although several hydrogel scaffolds have been designed for VF tissue engineering applications, none of these scaffolds mimic the VF's multi-layered biophysical properties. ${ }^{10,16,17,26}$ To better mimic the lamina propria structure, we used a layer-by-layer stacking technique to fabricate a tri-layered hydrogel scaffold that increased in stiffness from the top to deep layer. The hydrogel was synthesized using NorHA and crosslinked with both a non-degradable and an MMP-degradable crosslinker (Figure 2). The cell adhesive peptide RGD was added to promote cell adhesion and spreading. ${ }^{16,21}$ The overall degree of hydrogel degradability was tuned using the non-degradable to degradable crosslinker ratio. Partially-degradable hydrogels demonstrated high mesenchymal stem cell viability and spreading over seven days in vitro. Finally, the tri-layered hydrogel scaffold was successfully capable of vibrating and producing sound during proof-of-concept air flow studies. ${ }^{27}$ To the authors' knowledge, this work introduces the first tri-layered hydrogel scaffold that mimics the biophysical structure of the VF lamina propria and successfully vibrates during air flow testing.
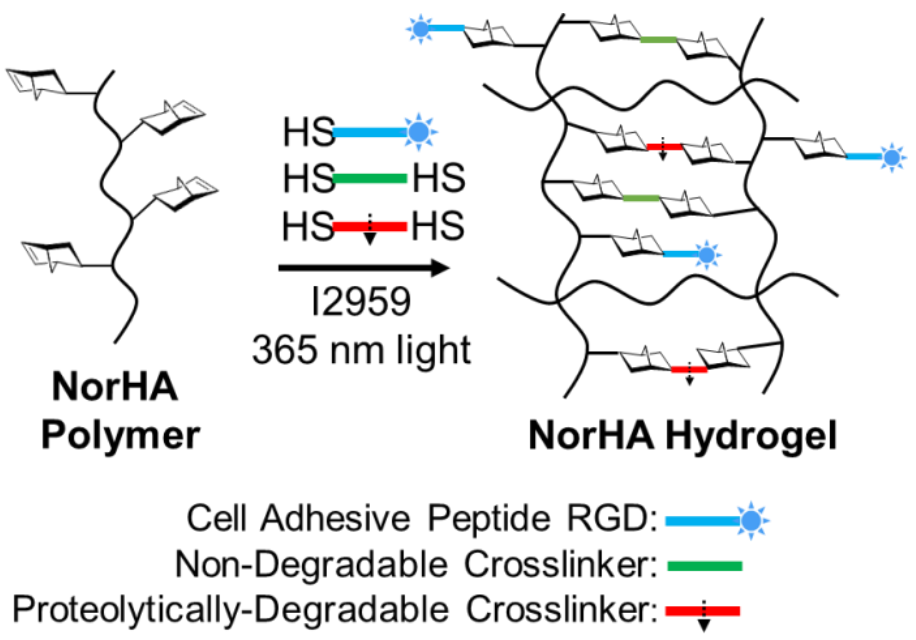

Figure 2: Norbornene-modified HA (NorHA) hydrogel synthesis: NorHA was crosslinked via UV light with a mixture of non-degradable and proteolytically-degradable crosslinkers to create a partially-degradable hydrogel. The cell adhesive peptide, RGD, was added to promote cell adhesion and spreading.

\section{Materials and Methods}

\section{Materials}

All chemicals were purchased from Sigma-Aldrich unless otherwise noted. 
Norbornene-Functionalized Hyaluronic Acid (NorHA) Synthesis

NorHA synthesis was adapted from previous research. ${ }^{28}$ Briefly, sodium hyaluronic acid (HA, Lifecore Biomedical, $60 \mathrm{kDa}$ ) was modified to tetrabutylammonium-hyaluronic acid (HA-TBA) using a Dowex $50 \mathrm{~W} \times 200$ ion exchange resin. HA-TBA was combined with 5-norbornene-2-methylamine (Tokyo Chemical Industry) and benzotriazole-1-yl-oxy-tris-(dimethylamino)-phosphonium hexafluorophosphate (BOP) and dissolved in anhydrous dimethyl sulfoxide (DMSO). Following reaction under nitrogen at room temperature for 4 hours, the resulting NorHA solution was purified via extensive dialysis, frozen, lyophilized, and analyzed using ${ }^{1} \mathrm{H}$ NMR (Figure S1).

\section{NorHA Hydrogel Formation}

Hydrogels were fabricated with polymer concentrations of $1.5,2,2.5,3$, and $4 \%$ w/v NorHA in phosphate buffer saline (PBS). NorHA with approximately $40 \%$ norbornene functionalization was dissolved in PBS containing 2 mM cell-adhesive peptide GCGYGRGDSPG (RGD, GenScript), a dithiol crosslinker, and $0.05 \%$ w/v photoinitiator (Irgacure-2959). Two dithiol crosslinkers were used: a matrix metalloproteinase (MMP)-degradable peptide GCRDVPM LSMRGGDRC (down arrow represents cleavage site, GenScript) and a non-degradable 1,4-dithiothreitol (DTT). Dithiol crosslinkers were added at a molar ratio of $0.4: 0.6: 1$ of MMP-degradable peptide to DTT to norbornene functional groups. The pre-gel solution was transferred into a custom cylindrical acrylic mold and crosslinked via ultraviolet (UV) light at $10 \mathrm{~mW} / \mathrm{cm}^{2}$ (Omnicure S1500, 320-390 nm) for 5 minutes.

\section{Tri-Layered NorHA Hydrogel Formation}

Individual pre-gel solutions were prepared as described in the NorHA Hydrogel Formation section. As shown in Figure 3A, tri-layered hydrogels were created using a layer-by-layer stacking technique. First, the pre-gel solution with the highest NorHA concentration was transferred into the cylindrical mold (deep layer) and partially crosslinked using UV light at $10 \mathrm{~mW} / \mathrm{cm}^{2}$ for 5 or 30 seconds. Next, the pre-gel solution with a medium NorHA concentration was transferred into the cylindrical mold (middle layer) and partially crosslinked using UV light at $10 \mathrm{~mW} / \mathrm{cm}^{2}$ for 5 or 30 seconds. Finally, the pre-gel solution with the lowest NorHA concentration was transferred into the cylindrical mold (top layer) and the entire tri-layered hydrogel was completely crosslinked using UV light at $10 \mathrm{~mW} / \mathrm{cm}^{2}$ for 
5 minutes. The tri-layered hydrogel consisted of the following NorHA concentrations in each layer: $1.5 \%$ (top), $2 \%$ (middle), and 2.5\% (deep) (Figure 3B). To visualize individual layers, red, green, or blue food coloring was added to each pre-gel solution prior to crosslinking (Figure 3C).

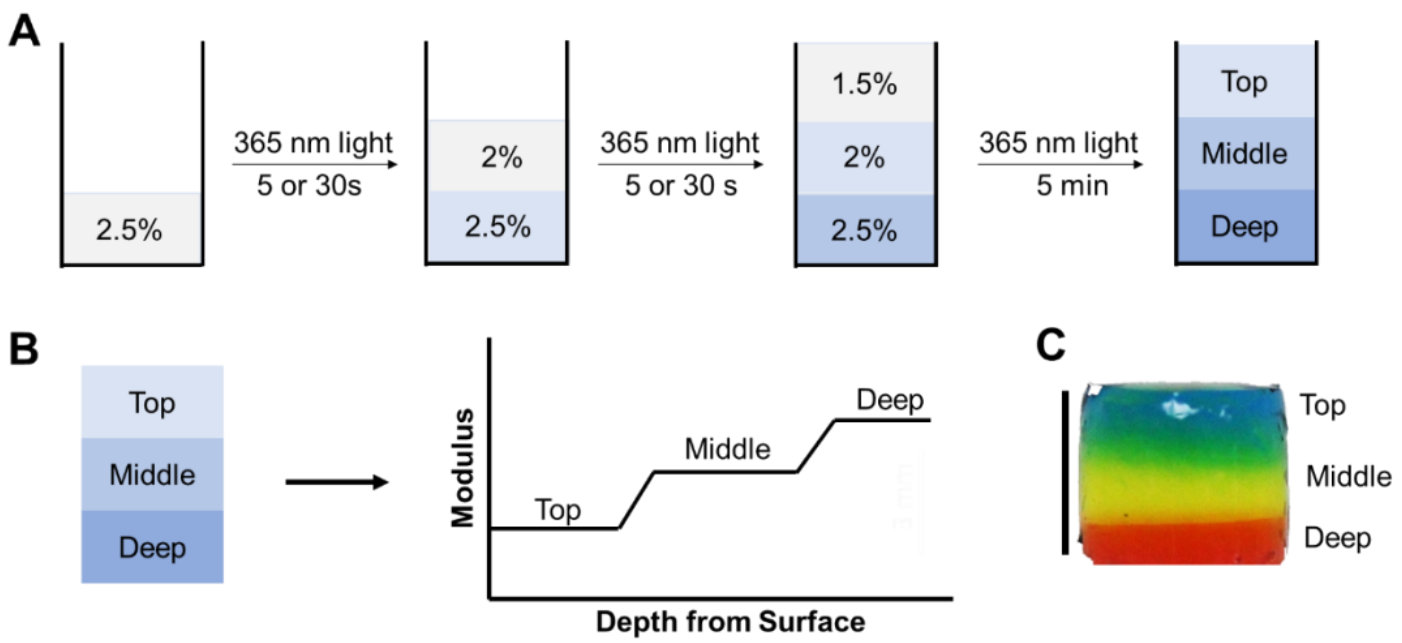

Figure 3: Tri-layered hydrogel scaffold fabrication using layer-by-layer stacking. (A) The highest HA concentration was deposited first (deep layer), followed by the intermediate HA concentration (middle layer), and the lowest HA concentration last (top layer). Each of the first two layers was partially crosslinked before adding the next layer and the entire tri-layered hydrogel was fully crosslinked for 5 minutes after the third layer was added. (B) Modulating HA concentration within each layer will enable control over layer modulus, with a higher modulus at higher HA concentrations. (C) Partial crosslinking during layer stacking resulted in interdigitated scaffold layers, scale $=3 \mathrm{~mm}$.

\section{Hydrogel Mechanical Property Characterization}

Shear and compression mechanical properties were assessed at room temperature immediately after gelation using cylindrical samples approximately $4.8 \mathrm{~mm}$ in diameter and $2 \mathrm{~mm}$ in height. Shear mechanical properties were assessed with increasing frequency using a dynamic mechanical analyzer (DMA) (Mettler Toldedo, DMA1) with a $10 \mathrm{~mm}$ diameter parallel plate geometry and a $2 \mathrm{~mm}$ gap distance. Oscillatory frequency sweeps were performed from $1-10 \mathrm{~Hz}$ with $1 \%$ strain. Shear storage and loss modulus was plotted as a function of frequency for each individual HA hydrogel concentration $(1.5,2,2.5,3$, and $4 \% \mathrm{w} / \mathrm{v}$ NorHA, $n=3)$.

A bench top mechanical testing machine (Instron Materials Testing System Series 5943, $50 \mathrm{~N}$ load cell) was used to test samples in unconfined compression. Compression was performed at $24 \%$ strain per minute and the compressive modulus was calculated from the initial linear region of the stress vs 
strain curve. The compressive modulus was determined for each individual HA hydrogel concentration $(1.5,2,2.5,3$, and $4 \% \mathrm{w} / \mathrm{v}$ NorHA) and the tri-layered hydrogel (three layers of $1.5,2$, and $2.5 \% \mathrm{w} / \mathrm{v}$ NorHA) $(\mathrm{n}=3)$.

\section{Cell Encapsulation and In Vitro Characterization}

Characterized human adipose-derived mesenchymal stem cells (hASCs) were obtained from subcutaneous fat from donors after receiving written informed consent and according to Mayo Clinic Institutional Review Board guidelines (female donor, IRB No. 14-001804, Figure S2). hASCs (passage 3) were suspended in $50 \mu \mathrm{L}$ of NorHA pre-gel solution $(1.5,2,2.5,3$, or $4 \% \mathrm{w} / \mathrm{v}$ NorHA) at a cell density of $2 \times 10^{6}$ cells $/ \mathrm{mL}$ to recapitulate the native cell density of the VF lamina propria. ${ }^{29}$ The pre-gel solution containing cells was crosslinked via UV light at $10 \mathrm{~mW} / \mathrm{cm}^{2}$ for $5 \mathrm{~min}$. Hydrogels with encapsulated hASCs were cultured in Dulbecco's Modified Eagle's Medium (DMEM) supplemented with $10 \%$ fetal bovine serum (FBS) at $37^{\circ} \mathrm{C}$ and $5 \% \mathrm{CO}_{2}$. After 1 and 7 days of in vitro cell culture, encapsulated cell viability and morphology was assessed $(n=4)$. Cell viability was evaluated using a live-dead assay (Biotum). Samples were imaged via confocal microscopy (Leica TCS SP5) and ImageJ was used to quantify viability as the percentage of live cells to total cells. Cell morphology was determined via fluorescein-5-maleimide (Life Technologies) and DAPI (Life Technologies) staining for the cell area and nucleus, respectively. Samples were imaged via confocal microscopy and ImageJ was used to quantify average cell number and area.

\section{Hydrogel Degradation}

Individual hydrogels $(1.5,2$, or $2.5 \% \mathrm{w} / \mathrm{v}$ NorHA) and the tri-layered hydrogel (three layers of $1.5,2$, and $2.5 \% \mathrm{w} / \mathrm{v}$ NorHA) were formed using a cylindrical acrylic mold. All hydrogel samples were $75 \mu \mathrm{L}$ in volume, where the tri-layered hydrogel had three layers of $25 \mu \mathrm{L}$ each. Partially-degradable hydrogels (40\% MMP-degradable peptide and 60\% non-degradable DTT crosslinks) were compared to fully degradable (100\% MMP-degradable peptide crosslinks) and non-degradable (100\% nondegradable DTT crosslinks) hydrogels. All samples were placed in $1 \mathrm{~mL}$ of Triton-Tris-Calcium buffer (TTC; $0.05 \%$ v/v Triton X 100, $50 \mathrm{mM}$ tris hydrochloride (Fisher Scientific), $1 \mathrm{mM}$ calcium chloride (Fisher Scientific), pH 7.4) with $20 \mathrm{U} / \mathrm{mL}$ of collagenase type II (Worthington Biochemical Corporation). The solution was changed every 24 hrs for 4 days and the collected degradation 
solution was stored at $-20^{\circ} \mathrm{C}$ until analysis. After 4 days, all remaining samples were degraded with 1 $\mathrm{mg} / \mathrm{mL}$ hyaluronidase in PBS. The amount of uronic acid, a degradation component of HA, was quantified using a modified uronic acid assay $(n=3) .{ }^{24}$

\section{Hydrogel Vibratory Evaluation}

As a proof of concept, the vibratory behavior of the tri-layered hydrogel scaffold (three layers of 1.5, 2, and $2.5 \% \mathrm{w} / \mathrm{v}$ NorHA) was evaluated using a custom-made air flow system. ${ }^{30}$ Tri-layered hydrogel scaffolds were molded into a 3D printed bracket as shown in Figure 4. Layers were molded in a stepwise manner and partially crosslinked with UV light for 5 seconds. The deep layer was molded first, followed by the middle, and then the top layer as depicted in Figure 4A. After the three layers were added to the bracket, the scaffold was fully crosslinked with UV light for 5 minutes. The top portion of the mold (black piece in Figure 4A) was removed following crosslinking to expose the superior surface of the scaffold. The molding procedure was designed to ensure the scaffolds were fully medialized, where the top layers of the tri-layered hydrogel scaffolds were facing each other and slightly touching when a pair of brackets were placed together (Figure 4C). A rectangular prism hydrogel geometry was chosen for its simplicity of molding and characterization. The rectangular prism was designed to approximate the vocal fold geometry in length, width, and thickness (Figure 4B). Similarly, the bracket shape was designed to approximate the laryngeal anatomy. Inferior to (below) the tri-layered hydrogel scaffold, the rigid bracket forms a converging shape to mimic the stiffer portion of the vocal folds that accelerates air flow through the glottis (i.e. the opening between the vocal folds).

The two brackets were held together with bolts and an o-ring was placed in the channel between the brackets to provide an airtight seal. Air flow was provided by compressed air and controlled via a mass flow controller (Whisper, Alicat Scientific) upstream of the scaffolds. Downstream of the mass flow controller, the air was humidified and heated with a Humicare D900 (ResMed) and HumiCare CondEx (ResMed), respectively (Figure 4D). Radiated sound measurements were recorded with a Bruel and Kjaer type 4192-L-001 microphone placed obliquely superior to (above) the scaffolds. A Photron Fastcam MC2 high-speed camera fit with a $17.5 \mathrm{~mm}$ lens was placed above the scaffolds to image the superior surface (Figure 4D). All data acquisition and air flow control were conducted with a 
custom LabView code running on a USB-6221 National Instruments data acquisition system.

Subglottal pressure, volume air flow rate, and microphone measurements were sampled at $50 \mathrm{kHz}$ and images were acquired at 2000 frames per second. Experiments were conducted with increasing air flow rate from 70 to $125 \mathrm{~mL} / \mathrm{s}$ in steps of $8 \mathrm{~mL} / \mathrm{s}$ to study the vibration of the tri-layered hydrogel scaffolds. Air flow rate was increased until phonation was observed and acquisition continued until irregular vibration of the scaffolds was observed. Data acquisition occurred for 0.5 seconds at a constant flow rate, and then flow rate was increased and the process repeated. In between runs of data collection, water was dripped onto the superior surface of the scaffolds to maintain hydration during testing.

Data processing was conducted with custom scripts in MATLAB. Power spectra and spectrograms were calculated from the radiated sound measurements recorded with the microphone. A window length of 8192 samples with $50 \%$ overlap was used giving a frequency resolution of $6.1 \mathrm{~Hz}$. Glottal area was computed using image thresholding to identify the open area between the two hydrogel scaffolds. Image scale calibration was performed using the known geometry of the 3D printed brackets.

A
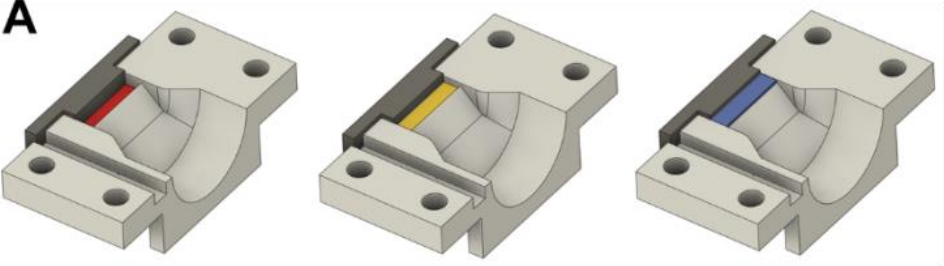

B
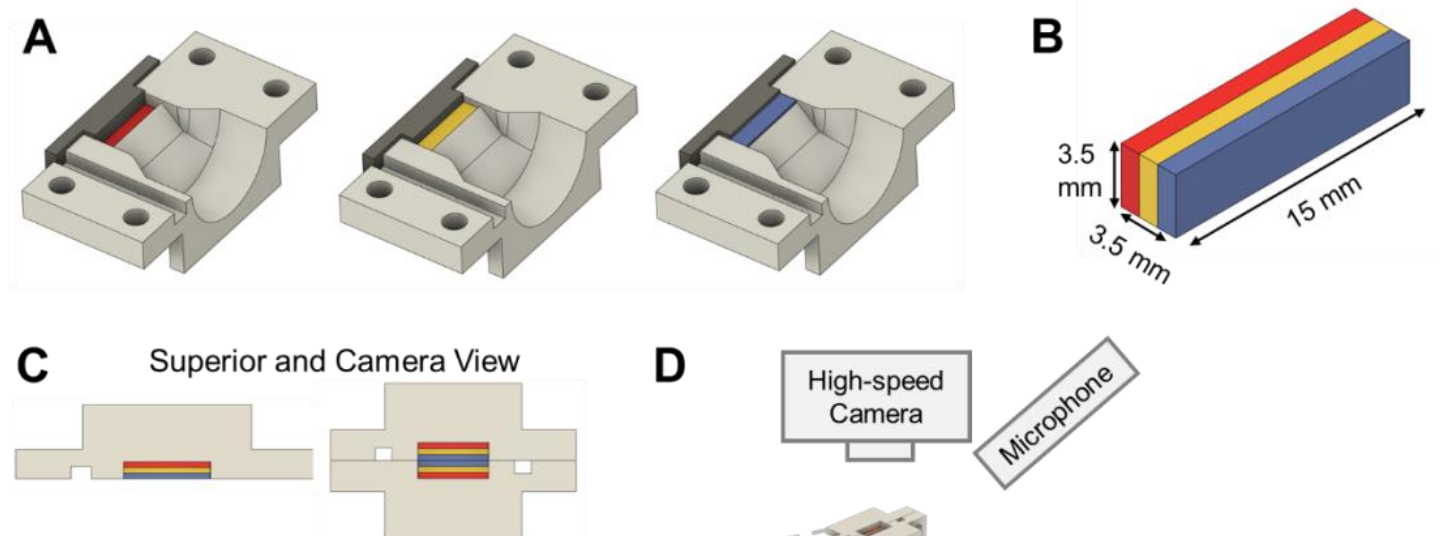

D

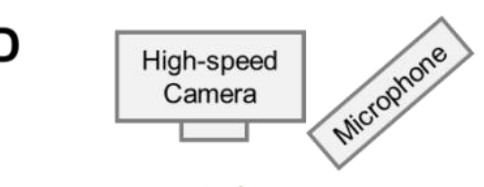

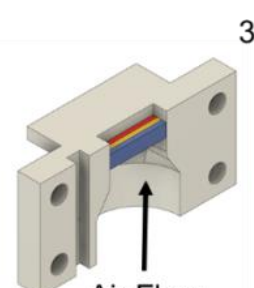

Air Flow
3D View

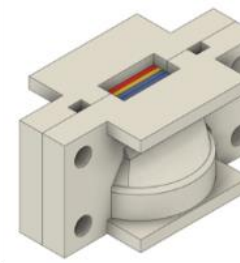

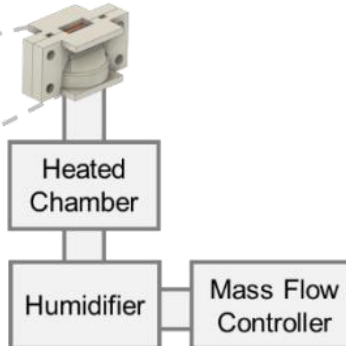

Compressed Air

Figure 4: Two tri-layered hydrogel scaffolds were molded within a 3D printed bracket in the shape of the larynx to evaluate vibratory behavior. (A) Each hydrogel layer was added in order of deep (left, red), middle (middle, yellow), top (right, blue) and partially crosslinked within the bracket. Once all three layers were added, the entire tri-layered hydrogel scaffold was fully crosslinked. (B) The hydrogel geometry is a rectangular prism 
with dimensions similar to the native vocal fold. (C) Two brackets, each containing a tri-layered hydrogel scaffold, were placed together to mimic the geometry of the vocal folds within the larynx. Arrow indicates direction of air flow through the bracket. (D) General schematic of the vibratory testing apparatus.

\section{Statistical Analysis}

All data was reported as the mean \pm standard deviation unless stated otherwise. For comparing results between two independent groups, a t-test was used to determine statistical significance, where $p<0.05$ was considered statistically significant. For tests that consisted of 1 independent variable with 3 or more treatment groups, a one-way ANOVA followed by Tukey's post hoc analysis was used to determine statistical significance, where $p<0.05$ was considered statistically significant.

\section{Results and Discussion}

Tri-layered hydrogels were successfully fabricated with increasing modulus through the layers The shear storage and loss modulus were determined for hydrogels with uniform concentrations of $1.5,2,2.5,3$, and $4 \% \mathrm{w} / \mathrm{v}$ NorHA using DMA. As a first estimation of hydrogel stability during vibration, hydrogels were tested with frequency sweeps of $1-10 \mathrm{~Hz} \cdot{ }^{31}$ The shear storage modulus increased with HA concentration and remained constant with increasing frequency up to $10 \mathrm{~Hz}$ (Figure 5A). The average storage modulus for the softest hydrogel with 1.5\% NorHA was $1.4 \mathrm{kPa}$, while the stiffest hydrogel with $4 \%$ NorHA was $12.6 \mathrm{kPa}$. The loss modulus was independent of concentration (Figure S3).

The static compressive modulus was also tested as a function of uniform hydrogel concentration (Figure 5B). Similar to the shear storage modulus, the compressive modulus increased with HA concentration, where the softest hydrogel with 1.5\% NorHA had an average modulus of $8.7 \mathrm{kPa}$ and the stiffest hydrogel with $4 \%$ NorHA had an average modulus of $74.9 \mathrm{kPa}$ (Figure $5 \mathrm{~B}$ ). The formation of a tri-layered hydrogel scaffold (three layers of $1.5,2$, and $2.5 \% \mathrm{w} / \mathrm{v}$ NorHA) was formed via layerby-layer stacking (Figure 3). To confirm the successful formation of a tri-layered hydrogel, individual hydrogel layers were visualized by incorporating dye into the top (blue), middle (yellow), and deep (red) pre-gel solutions prior to crosslinking (Figure 3C). Tri-layered hydrogels were tested under compression using two different partial crosslinking times between layers: 5 and 30 seconds (Figure 5C). For both partial crosslinking times, the entire tri-layered hydrogel was completely crosslinked for 5 minutes prior to testing. The compression modulus for the tri-layered hydrogel scaffolds was 
approximately $14 \mathrm{kPA}$ and was comparable to the $2 \% \mathrm{HA}$ hydrogel (middle layer). Interestingly, the modulus for the shorter partial crosslinking time was slightly higher compared to the higher partial crosslinking time; however, this difference was not statistically significant. Other studies have noted shorter partial crosslinking times may increase pre-gel solution diffusion into neighboring layers and result in improved interdigitation between layers. ${ }^{32}$ The tri-layered hydrogel was stiffer compared to the native VF lamina propria by an order of magnitude. ${ }^{7}$ However, ex vivo mechanical testing is not a precise assessment of the VF's mechanical functionality in vivo. Further, the partially-degradable hydrogel scaffold will soften over time as the MMP-sensitive crosslinks degrade. An in vivo study with a more complete analysis of the dynamic mechanical properties and functionality of the tri-layered hydrogel is needed for future studies. Nonetheless, these results confirm that layer-by-layer stacking with increasing concentration will produce a stable tri-layered hydrogel suitable to undergo proof of concept vibratory testing. ${ }^{1}$
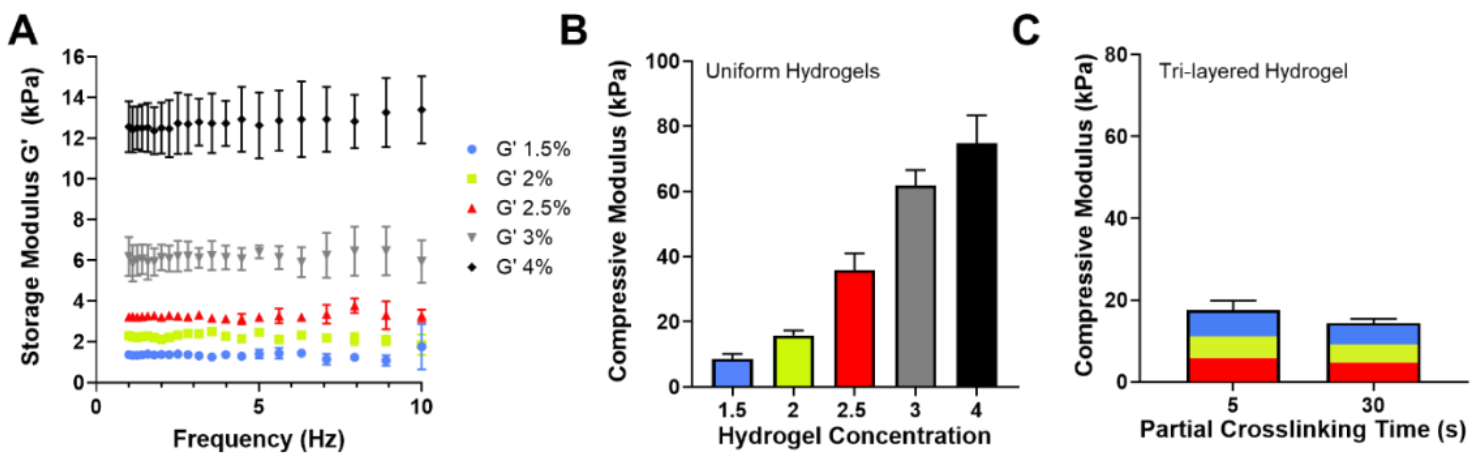

Figure 5: HA hydrogel modulus is highly dependent on HA concentration. (A) Dynamic shear storage modulus from $1-10 \mathrm{~Hz}$ and $(\mathrm{B})$ static compressive modulus for uniform $\mathrm{HA}$ concentrations between $1.5-4 \% \mathrm{w} / \mathrm{v}$ NorHA. (C) Static compressive modulus for the tri-layered hydrogel (three layers of 1.5, 2, and 2.5\% w/v NorHA) with partial crosslinking times of 5 or 30 seconds.

\section{Cell viability is high for most hydrogel crosslinking densities}

To evaluate the viability of hASCs encapsulated within HA hydrogels, a live/dead assay was performed for hydrogels with uniform concentrations of $1.5,2,2.5,3$, and 4\% w/v NorHA at 1 and 7 days of in vitro cell culture. After 1 day of culture, all hydrogel concentrations exhibited high cell viability above $80 \%$ (Figure 6). On day 7 , all hydrogel groups continued to maintain high cell viability above $80 \%$ except for the highest concentration at $4 \%$ NorHA. The average viability for $4 \%$ NorHA hydrogels was slightly lower at $65 \%$ after 7 days of cell culture. Hydrogels with higher polymer concentrations will have a correspondingly higher crosslink density and smaller mesh size between 
individual crosslinks. Research has demonstrated nutrient diffusion is impeded within hydrogels with small mesh sizes. ${ }^{33,34}$ Thus, lower cell viability at the highest HA concentration with the smallest mesh size may be due to reduced nutrient diffusion. ${ }^{35}$ The impact of UV-initiated and radical-mediated crosslinking reactions on cell viability is also an ongoing concern within the biomaterials community. Recent research has reported near-UV light $(300-400 \mathrm{~nm})$ does not negatively influence mesenchymal stem cell behavior for low exposure times similar to those used here. ${ }^{36}$ However, free radicals produced during UV exposure has been shown to negatively impact mesenchymal stem cell viability. ${ }^{37-39}$ Higher free radical density within higher HA concentration hydrogels during the crosslinking step may further explain the slight decrease in cell viability observed at 7 days in $4 \%$ NorHA hydrogels.

A

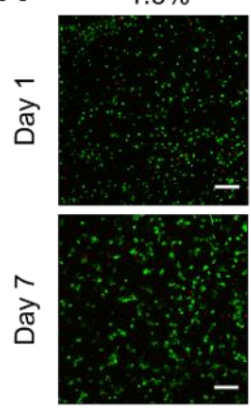

$2 \%$
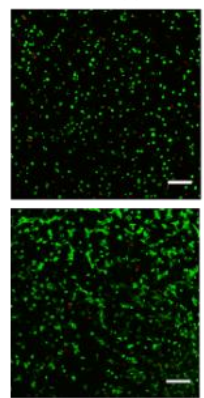

$2.5 \%$
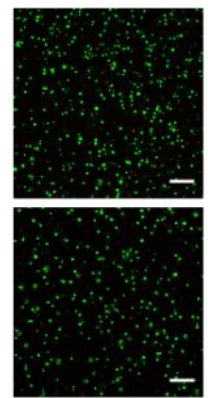

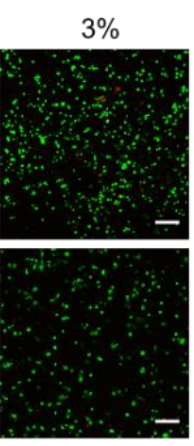

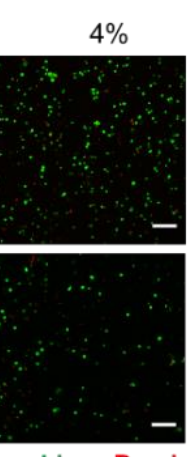

Live Dead

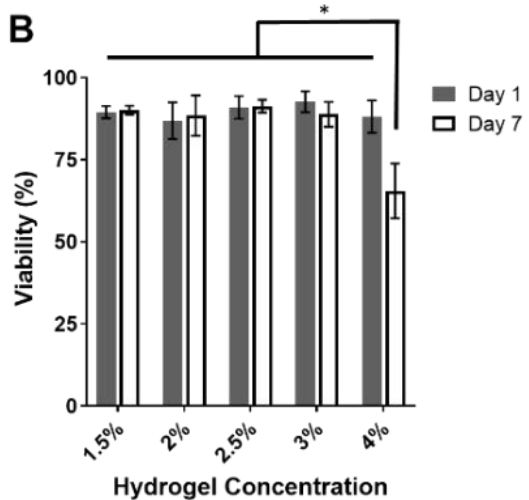

Figure 6: Hydrogels maintained high cell viability for $\mathbf{1 . 5 - 3} \%$ NorHA after 7 days. (A) Live/dead staining and (B) corresponding cell viability after 1 and 7 days of in vitro cell culture as a function of HA concentration (scale = $200 \mu \mathrm{m})$. *Indicates statistical significance $(\mathrm{p}<0.05)$.

\section{Increased cell spreading occurs in hydrogels with lower crosslinking density}

To induce 3D cell spreading, hASCs were encapsulated within a partially-degradable NorHA hydrogel containing $40 \%$ proteolytically-degradable crosslinks and $60 \%$ non-degradable crosslinks. Cell morphology was analyzed after 1 and 7 days of in vitro cell culture for hydrogels with uniform concentrations of $1.5,2,2.5,3$, and $4 \%$ w/v NorHA (Figure 7). After 1 day of culture, encapsulated cells were rounded with minimal spreading and an average cell area of approximately $400 \mu \mathrm{m}^{2}$ for all hydrogel groups. After 7 days of culture, cells encapsulated within 1.5-2.5\% NorHA hydrogels were more spread compared to day 1 with an average cell area of approximately $580 \mu \mathrm{m}^{2}$. However, hASCs encapsulated within 3-4\% NorHA hydrogels did not exhibit increased spreading after one week of culture. Previous work has demonstrated the importance of incorporating proteolytically- 
degradable crosslinks into hydrogels to allow cells to degrade their local environment and enable 3D cell spreading. ${ }^{22}$ In this work, partially-degradable hydrogels with a higher polymer concentration have a higher crosslink density and, particularly, a higher density of non-degradable crosslinks. These nondegradable crosslinks limit the ability of the encapsulated cells to degrade the surrounding material and may impede 3D cell spreading. Due to higher cell viability, increased cell spreading, and mechanical properties that are closest to the VF lamina propria, ${ }^{7}$ a tri-layered hydrogel scaffold with three layers of $1.5,2$, and $2.5 \% \mathrm{w} / \mathrm{v}$ NorHA was chosen for all future experiments.
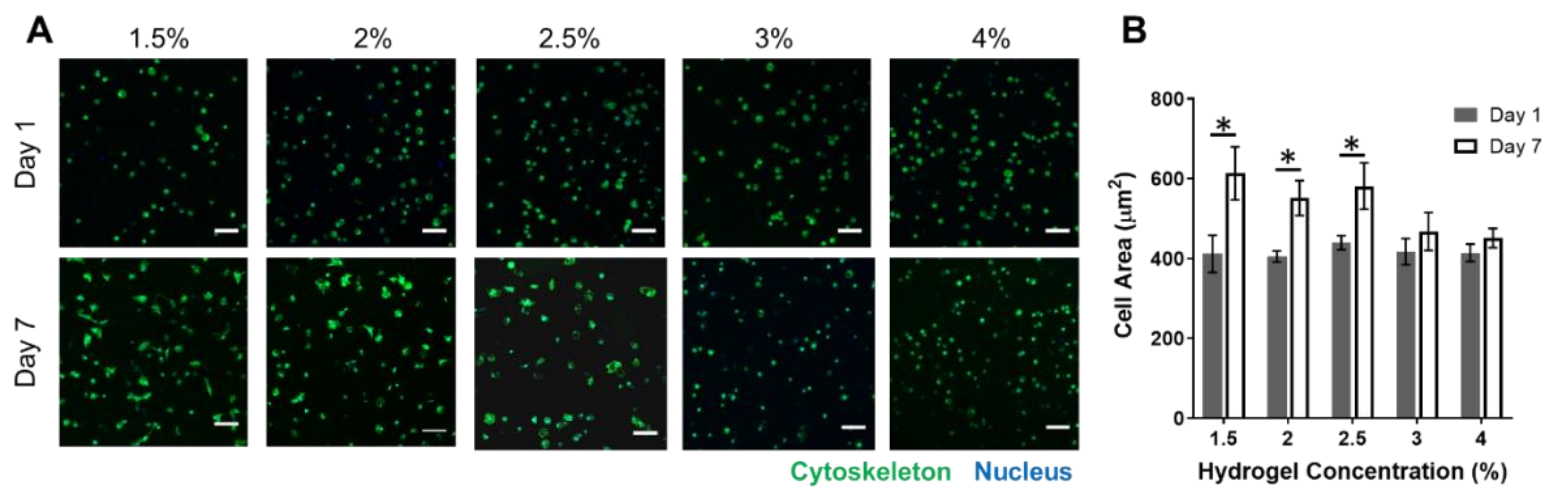

Figure 7: Hydrogels with lower crosslinking density enabled cell spreading in 3D. (A) Cytoskeletal and nuclear staining with (B) corresponding cell area after 1 and 7 days of in vitro culture as a function of HA concentration $($ scale $=100 \mu \mathrm{m})$. ${ }^{*}$ Indicates statistical significance $(p<0.05)$.

\section{Hydrogel degradation is dependent on the crosslink type}

To assess hydrogel degradation kinetics, hydrogels were incubated in collagenase and HA degradation was measured using a uronic acid assay. Collagenase contains several different proteases, including MMPs, and was chosen for the degradation study as MMPs are the primary enzymes associated with ECM remodeling in vivo. ${ }^{40,41}$ The degradation behavior was evaluated for individual hydrogels with uniform concentrations of $1.5,2$, or $2.5 \% \mathrm{w} / \mathrm{v}$ NorHA, as well as the trilayered hydrogel scaffold (three layers of 1.5, 2, and 2.5\% w/v NorHA) (Figure 8). Partially-degradable hydrogels were fabricated with $40 \%$ proteolytically-degradable crosslinks and $60 \%$ non-degradable crosslinks. Partially-degradable hydrogels were compared to fully-degradable (100\% proteolyticallydegradable crosslinks) and non-degradable (100\% non-degradable crosslinks) hydrogels. Fullydegradable hydrogels completely degraded within one day. Non-degradable hydrogels lost around $20 \%$ of the initial hydrogel's mass, likely due to some of the HA polymer chains not being fully crosslinked into the polymer network. Partially-degradable hydrogels degraded at rates in between 
the non-degradable and fully-degradable hydrogels with a mass loss of approximately $50-65 \%$ after 4 days. These results demonstrate partially-degradable HA hydrogels remain stable even after collagenase exposure. While protease-degradability is critical for promoting cell spreading and viability, a longer-term hydrogel structure is clinically desirable to enable functionality (e.g. swallowing, coughing, speaking, etc.) during the tissue regeneration process. In vitro degradation using collagenase is not a perfect representation of the in vivo environment. Further experimentation in vivo is required to determine the scaffold degradation and new tissue formation kinetics, as well as how these changes impact scaffold mechanical properties.
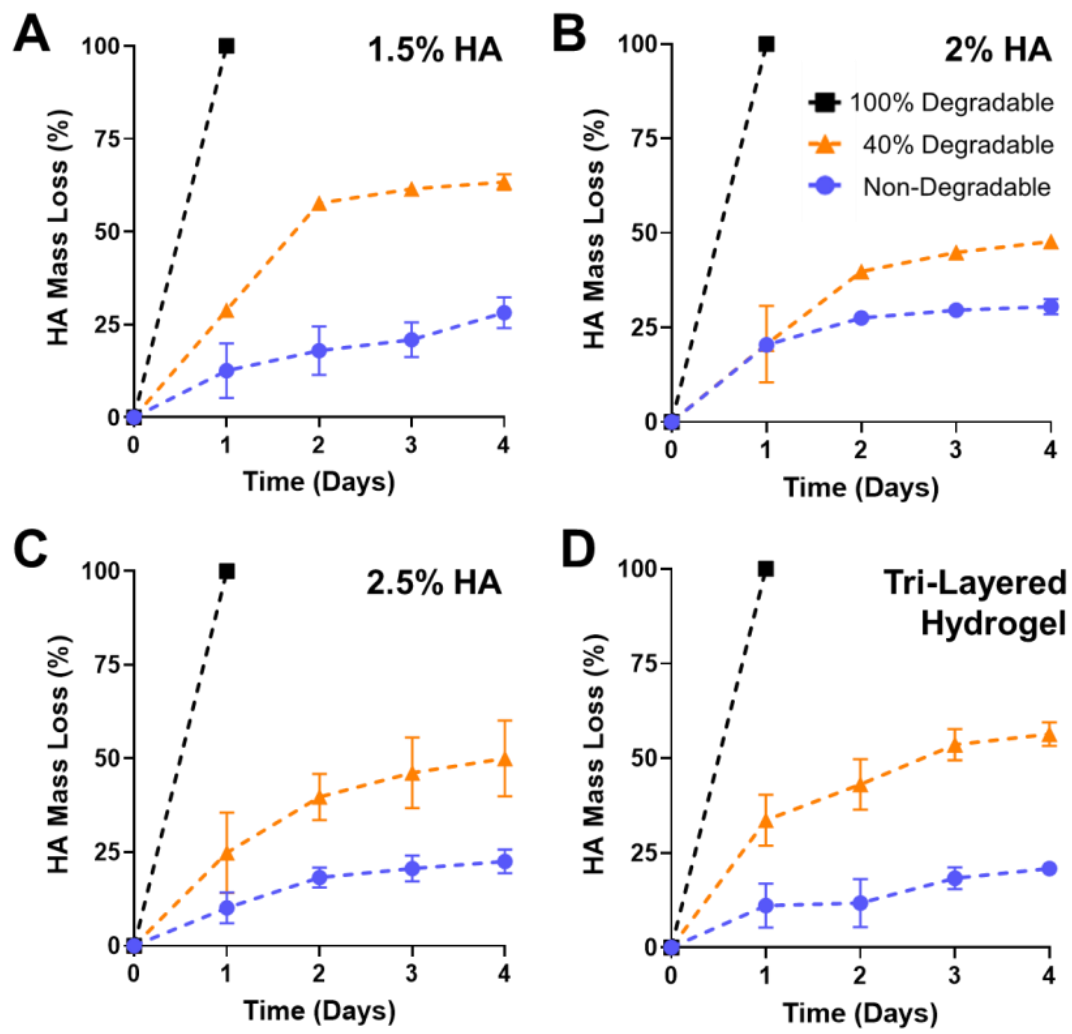

Figure 8: Partially-degradable hydrogels were responsive to collagenase while maintaining part of the polymer network for stability. HA mass loss during exposure to collagenase for uniform hydrogels with (A) 1.5 , (B) 2, and (C) 2.5\% w/v NorHA, as well as (D) the tri-layered hydrogel scaffold. Mass loss was measured using a uronic acid assay and partially-degradable hydrogels were compared to non-degradable and fully-degradable hydrogels.

\section{Tri-layered hydrogel scaffold successfully vibrates under air flow}

Two tri-layered hydrogel scaffolds were molded adjacently into a 3D bracket in the approximate shape of the larynx. To mimic the multi-layered structure of the VF lamina propria, the hydrogels were 
fabricated with the stiffest (deep) layer on the bracket surface and the softest (top) layer facing the adjacent hydrogel.

During air flow, the tri-layered hydrogel scaffolds opened and closed as visualized through high-speed imaging at an air flow rate of $104 \mathrm{~mL} / \mathrm{s}$ (Figure 9). A single vertical line of pixels across the glottal area is depicted as a function of time during air flow, which displays the vibratory wave that occurs during phonation (Figure 9B). The medial (i.e. middle) surface between the two hydrogel scaffolds was observed to open and close in response to air flow. The glottal area (i.e. the dark region between the two scaffolds) in Figure 9C shows the tri-layered hydrogel scaffolds open with a maximum glottal area of approximately $4 \mathrm{~mm}^{2}$ and close with a minimum area of approximately $1 \mathrm{~mm}^{2}$. Representative images of the opening and closing of the tri-layered hydrogel scaffolds are shown in Figure 9D.

Sound produced during hydrogel vibration was measured using a microphone. A spectrogram of the sound pressure level (SPL) in decibels is shown as a function of frequency and air flow rate (Figure 10A). The air flow rate through the tri-layered hydrogel scaffolds was increased from 70 to $125 \mathrm{~mL} / \mathrm{s}$ in steps of $8 \mathrm{~mL} / \mathrm{s}$. As the air flow rate increased, scaffold vibration onset occurred at roughly $80 \mathrm{~mL} / \mathrm{s}$ with a fundamental frequency of vibration at roughly $150 \mathrm{~Hz}$. The high SPL corresponding to the fundamental frequency is noted with a red asterisk and its harmonics are noted with black asterisks in Figure 10A. Subharmonics appear at air flow rates above $95 \mathrm{~mL} / \mathrm{s}$ and are noted with black plus signs in Figure 10A. Figure 10B further illustrates this behavior by comparing the spectrum at two different air flow rates. Only the harmonics $\left(^{*}\right)$ are present at the $95 \mathrm{~mL} / \mathrm{s}$ air flow rate. Harmonics $\left(^{*}\right)$ and subharmonics (+) appear in the spectrum at the $115 \mathrm{~mL} / \mathrm{s}$ air flow rate. Normal human voice spectra have many harmonics of the fundamental frequency. The presence of subharmonics in this data indicates some non-linear behaviors that may be undesirable for a healthy sounding voice. For reference, average air flow rates in human phonation have been measured in the range of 140-235 $\mathrm{mL} / \mathrm{s}$ for a soft, normal, and loud voice in male and female subjects. ${ }^{42}$ The presence of subharmonics below these normal human conditions indicates further refinement of the scaffold structure should be considered so that adequate vibration and sound production is achieved over a wider range of conditions. Nonetheless, the tri-layered hydrogel scaffolds successfully vibrated and produced sound 
during the air flow studies which is an important first step in biomimetic scaffold design for vocal fold regeneration.

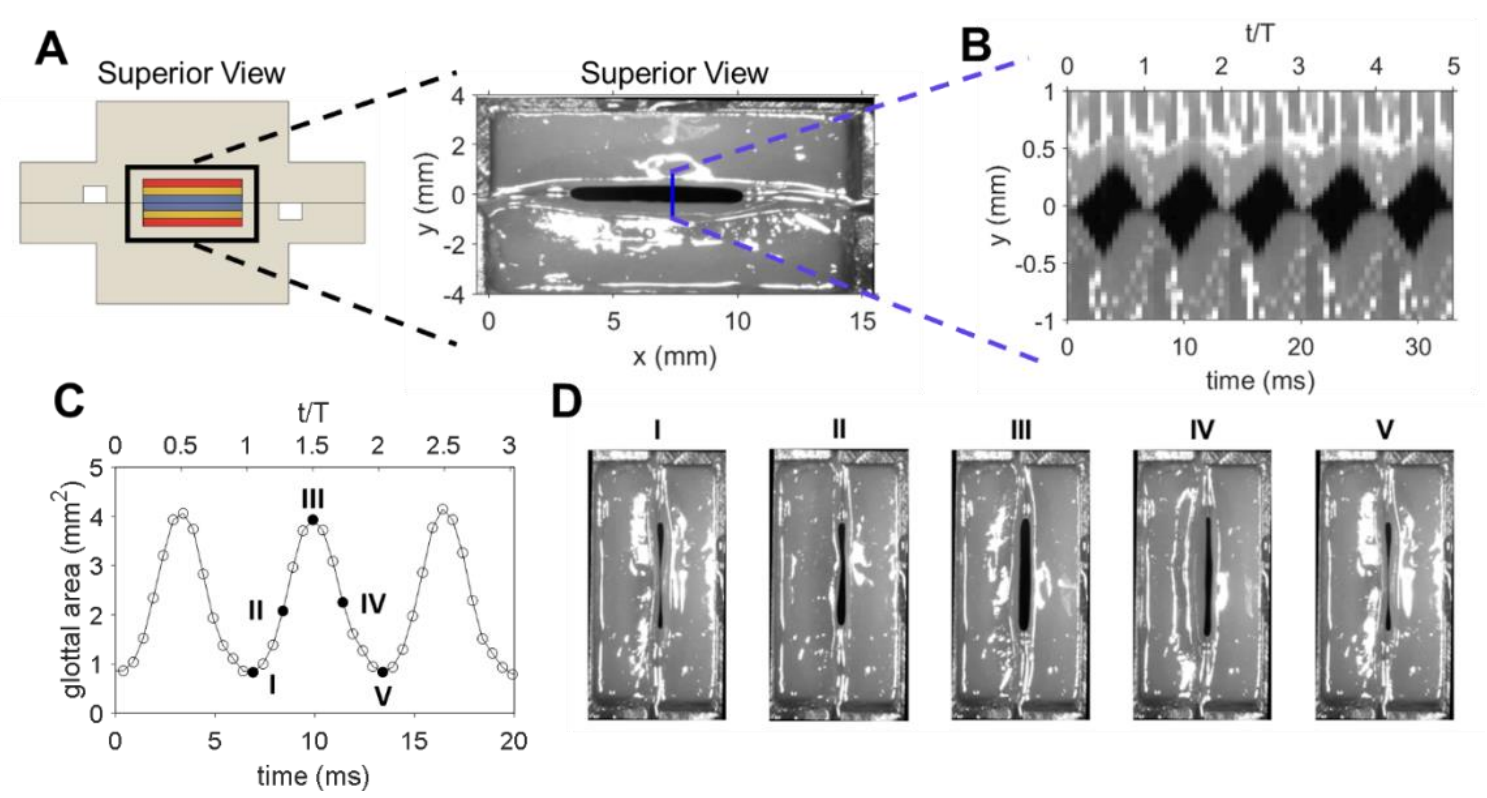

Figure 9: Tri-layered hydrogel scaffolds successfully vibrated under air flow. (A) Schematic of the superior surface of the tri-layered hydrogel scaffolds within the bracket (left) and image of the superior surface of the trilayered hydrogel scaffolds during air flow testing (right). (B) Vibratory waves during air flow were visualized by tracking the pixels in a single vertical line, where the blue line in A represents the approximate location. (C) Glottal area as a function of time with (D) five time points (black circles in C) selected to show the opening and closing of the glottal area between the tri-layered hydrogel scaffolds.

A

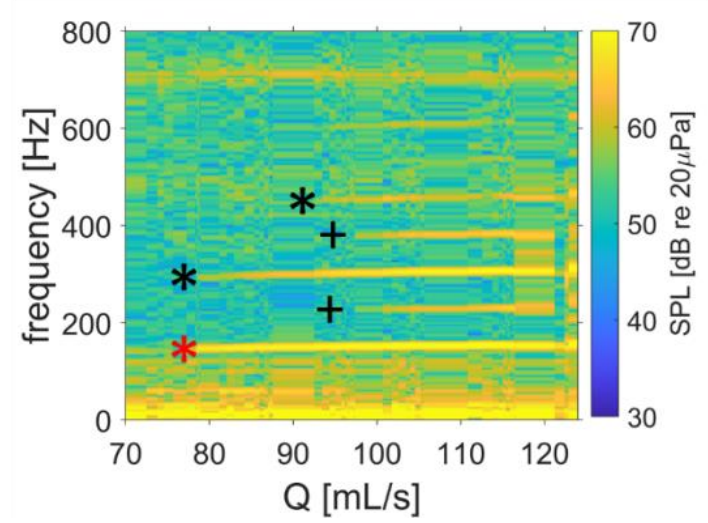

B

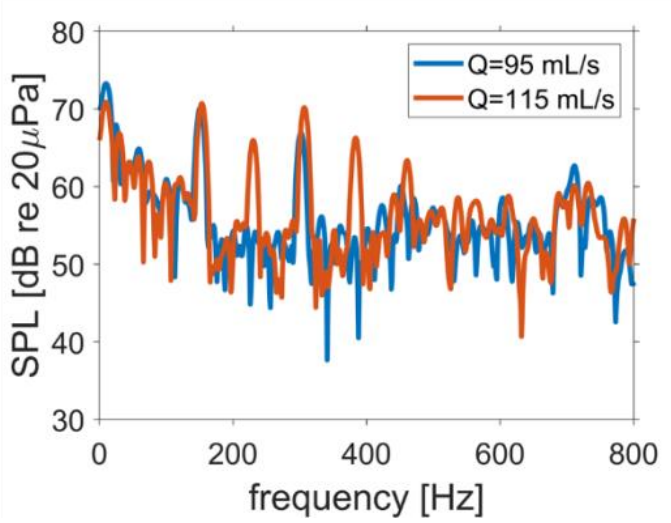

Figure 10: Spectral analysis demonstrated successful phonation by the vibrating tri-layered hydrogels. (A) Spectrogram with sound pressure level (SPL) and frequency as a function of air flow rate (Q). (B) SPL in decibels $(\mathrm{dB})$ as a function of frequency for two air flow rates, 95 and $115 \mathrm{~mL} / \mathrm{s}$.

\section{Conclusion}

Current VF restoration strategies do not adequately restore function following VF scarring. To restore proper function, it is critical to mimic the heterogenous biophysical structure of the VF lamina propria 
which is responsible for vibration. In this study, we successfully fabricated a tri-layered NorHA hydrogel scaffold that mimics the multi-layered structure within the native VF lamina propria. The trilayered hydrogel was formed via layer-by-layer stacking with increasing stiffness from the top layer (softest) to the deep layer (stiffest). The hydrogels were designed to be partially-degradable using a combination of proteolytically-degradable and non-degradable crosslinks. Partially-degradable hydrogels offered the advantage of increased stability following exposure to proteases, while still enabling high cell viability and increased cell spreading in 3D hydrogels. Additionally, the tri-layered hydrogel scaffold (three layers of $1.5,2$, and $2.5 \%$ w/v NorHA) successfully phonated (produced sound via vibration) during air flow studies. As a result, this work represents an important first step in the design of multi-layered hydrogel scaffolds that mimic the biophysical structure of the lamina propria for VF tissue engineering. Future work will evaluate tri-layered HA hydrogels using an in vivo animal model to further evaluate hydrogel stability and functionality. Furthermore, many tissues within the body are composed of heterogeneous, layered, biophysical structures, where this approach would be widely applicable.

\section{Acknowledgements}

Funding for this project was provided by the Mayo Clinic and ASU Alliance Collaborative Research Seed Grant. Instrumentation support was provided in part by ASU's Advanced Light Microscopy Core Facility, Regenerative Medicine Bioimaging Core Facility, and the Magnetic Resonance Research Center. Finally, the authors would like to thank Fallon Fumasi for assistance with polymer synthesis. 


\section{Graphical Abstract, TOC Figure}

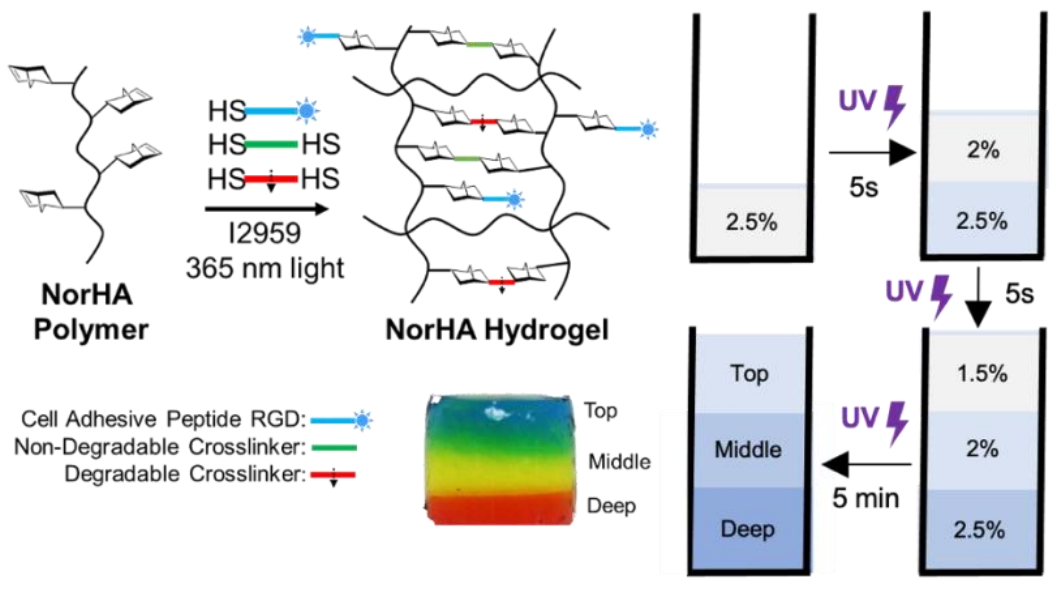

\section{References}

1. Lott, D. G. \& Janus, J. R. Tissue engineering for otorhinolaryngology-head and neck surgery. Mayo Clin. Proc. 89, 1722-1733 (2014).

2. Cohen, S. M., Kim, J., Roy, N., Asche, C. \& Courey, M. Prevalence and causes of dysphonia in a large treatment-seeking population. Laryngoscope 122, 343-348 (2012).

3. Svistushkin, M. V. et al. Collagen fibrillar structures in vocal fold scarring and repair using stem cell therapy: a detailed histological, immunohistochemical and atomic force microscopy study. J. Microsc. 274, 55-68 (2019).

4. Kobayashi, T. et al. Drug delivery system of basic fibroblast growth factor using gelatin hydrogel for restoration of acute vocal fold scar. Auris Nasus Larynx 44, 86-92 (2017).

5. Fishman, J. M. et al. Stem cell approaches for vocal fold regeneration. Laryngoscope 126, 1865-1870 (2016).

6. Rohlfs, A. K. et al. The anisotropic nature of the human vocal fold: An ex vivo study. Eur. Arch. Oto-Rhino-Laryngology 270, 1885-1895 (2013).

7. Miri, A. K. Mechanical characterization of vocal fold tissue: A review study. J. Voice 28, 657667 (2014).

8. Hansen, J. K. \& Thibeault, S. L. Current understanding and review of the literature: Vocal fold scarring. J. Voice 20, 110-120 (2006).

9. Ward, P. D., Thibeault, S. L. \& Gray, S. D. Hyaluronic acid: Its role in voice. J. Voice 16, 303309 (2002).

10. Walimbe, T., Panitch, A. \& Sivasankar, P. M. A Review of Hyaluronic Acid and Hyaluronic 
Acid-based Hydrogels for Vocal Fold Tissue Engineering. J. Voice 31, 416-423 (2017).

11. Rousseau, B. et al. Characterization of chronic vocal fold scarringin a rabbit model. J. Voice 18, 116-124 (2004).

12. Reyes Valenzuela, A. et al. Polymeric Microspheres Containing Human Vocal Fold Fibroblasts for Vocal Fold Regeneration. Laryngoscope (2020) doi:10.1002/lary.29118.

13. O'Brien, F. J. Biomaterials \& scaffolds for tissue engineering. Mater. Today 14, 88-95 (2011).

14. Choi, J. W. et al. Small intestine submucosa and mesenchymal stem cells composite gel for scarless vocal fold regeneration. Biomaterials 35, 4911-4918 (2014).

15. Hiwatashi, N. et al. The efficacy of a novel collagen-gelatin scaffold with basic fibroblast growth factor for the treatment of vocal fold scar. J. Tissue Eng. Regen. Med. 11, 1598-1609 (2017).

16. Walimbe, T., Calve, S., Panitch, A. \& Sivasankar, M. P. Incorporation of types I and III collagen in tunable hyaluronan hydrogels for vocal fold tissue engineering. Acta Biomater. 87, 97-107 (2019).

17. Hahn, M. S., Teply, B. A., Stevens, M. M., Zeitels, S. M. \& Langer, R. Collagen composite hydrogels for vocal fold lamina propria restoration. Biomaterials 27, 1104-1109 (2006).

18. Li, L. et al. Tissue engineering-based therapeutic strategies for vocal fold repair and regeneration. Biomaterials 108, 91-110 (2016).

19. Choi, J. S. et al. Preservation of viscoelastic properties of rabbit vocal folds after implantation of hyaluronic acid-based biomaterials. Otolaryngol. - Head Neck Surg. (United States) 147, $515-521$ (2012).

20. Lin, C. C., Ki, C. S. \& Shih, H. Thiol-norbornene photoclick hydrogels for tissue engineering applications. J. Appl. Polym. Sci. 132, 1-11 (2015).

21. Wade, R. J., Bassin, E. J., Gramlich, W. M. \& Burdick, J. A. Nanofibrous Hydrogels with Spatially Patterned Biochemical Signals to Control Cell Behavior. Adv. Mater. 27, 1356-1362 (2015).

22. Khetan, S. et al. Degradation-mediated cellular traction directs stem cell fate in covalently crosslinked three-dimensional hydrogels. Nat. Mater. 12, 458-465 (2013).

23. Liao, H. et al. Influence of hydrogel mechanical properties and mesh size on vocal fold fibroblast extracellular matrix production and phenotype. Acta Biomater. 4, 1161-1171 (2008). 
24. Khetan, S., Katz, J. S. \& Burdick, J. A. Sequential crosslinking to control cellular spreading in 3-dimensional hydrogels. Soft Matter 5, 1601-1606 (2009).

25. Shapiro, S. D. Matrix metalloproteinase degradation of extracellular matrix: Biological consequences. Curr. Opin. Cell Biol. 10, 602-608 (1998).

26. Park, H. et al. Three-dimensional hydrogel model using adipose-derived stem cells for vocal fold augmentation. Tissue Eng. - Part A 16, 535-543 (2010).

27. Loebel, C. \& Burdick, J. A. Cell Stem Cell Engineering Stem and Stromal Cell Therapies for Musculoskeletal Tissue Repair. Stem Cell 22, 325-339 (2018).

28. Vega, S. L. et al. Combinatorial hydrogels with biochemical gradients for screening 3D cellular microenvironments. Nat. Commun. 9, 1-10 (2018).

29. Long, J. L., Zuk, P., Berke, G. S. \& Chhetri, D. K. Epithelial differentiation of adipose-derived stem cells for laryngeal tissue engineering. Laryngoscope 120, 125-131 (2010).

30. Chhetri, D. K., Neubauer, J. \& Berry, D. A. Neuromuscular control of fundamental frequency and glottal posture at phonation onset. J. Acoust. Soc. Am. 131, 1401-1412 (2012).

31. Wittenberg, T., Tigges, M., Mergell, P. \& Eysholdt, U. Functional imaging of vocal fold vibration: Digital multislice high-speed kymography. J. Voice 14, 422-442 (2000).

32. Ko, H. et al. A simple layer-stacking technique to generate biomolecular and mechanical gradients in photocrosslinkable hydrogels. Biofabrication 11, (2019).

33. Cha, C., Kim, S. Y., Cao, L. \& Kong, H. Decoupled control of stiffness and permeability with a cell-encapsulating poly(ethylene glycol) dimethacrylate hydrogel. Biomaterials 31, 4864-4871 (2010).

34. Schmidt, J. J., Rowley, J. \& Hyun, J. K. Hydrogels used for cell-based drug delivery. J. Biomed. Mater. Res. - Part A 87, 1113-1122 (2008).

35. Rehmann, M. S. et al. Tuning and Predicting Mesh Size and Protein Release from Step Growth Hydrogels. Biomacromolecules 18, 3131-3142 (2017).

36. Ruskowitz, E. R. \& Deforest, C. A. Proteome-wide Analysis of Cellular Response to Ultraviolet Light for Biomaterial Synthesis and Modification. ACS Biomater. Sci. Eng. 5, 2111-2116 (2019).

37. Bahney, C. S. et al. Visible light photoinitiation of mesenchymal stem cell-laden bioresponsive hydrogels. Eur. Cells Mater. 22, 43-55 (2011). 
38. Fedorovich, N. E. et al. The effect of photopolymerization on stem cells embedded in hydrogels. Biomaterials 30, 344-353 (2009).

39. Williams, C. G., Malik, A. N., Kim, T. K., Manson, P. N. \& Elisseeff, J. H. Variable cytocompatibility of six cell lines with photoinitiators used for polymerizing hydrogels and cell encapsulation. Biomaterials 26, 1211-1218 (2005).

40. Lu, P., Takai, K., Weaver, V. M. \& Werb, Z. Extracellular Matrix degradation and remodeling in development and disease. Cold Spring Harb. Perspect. Biol. 3, 1-24 (2011).

41. Streuli, C. Extracellular matrix remodelling and cellular differentiation. Curr. Opin. Cell Biol. 11, $634-640$ (1999).

42. Holmberg, E. B., Hillman, R. E. \& Perkell, J. S. Glottal airflow and transglottal air pressure measurements for male and female speakers in soft, normal, and loud voice. J. Acoust. Soc. Am. 84, 511-529 (1988). 